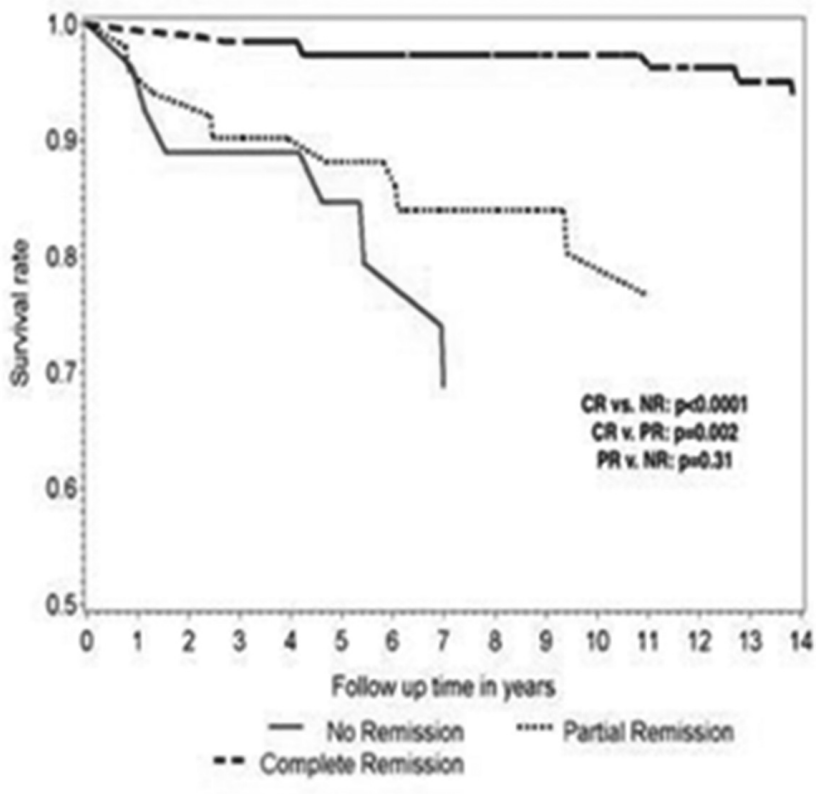

Abstract 219 Figure 1 Kaplan Meier analysis of long term outcome of low eGFR ( $<15 \mathrm{~mL} / \mathrm{min})$ among patients who acheived $C R, P R$ or NR at 2 years of $L N$ diagnosis

from 81 patients with SLE.We examined anti-dsDNA level, clinical features and kidney laboratory profile in all patient. The obtained data were statistically analysed.

Results 81 SLE patients with mean level of anti-dsDNA $294 \mathrm{IU} / \mathrm{ml}$ (6.1-1317). There is no significant relationship between increased level of Anti-dsDNA with other clinical manifestations $(p>0.05)$. There are significant relationships between increased level of Anti-dsDNA with ureum level $(\mathrm{p}=0.016), \quad$ thrombocytopenia $\quad(\mathrm{p}=0.001), \quad$ leucopenia $(\mathrm{p}=0.006)$, kidney disorder $(\mathrm{p}=0.049)$ and urine protein $(\mathrm{p}=0.028)$.Arthritis is the most frequent clinical manifestation (96.3\%) from this study followed by malar rash $(77.8 \%)$ and photo sensitivity $(40.7 \%)$.

Conclusions Elevated anti-ds DNA level was not correlated with clinical symptoms but there is significant correlation with haematology disorder and kidney laboratory profiles of SLE patients.

\section{SELECTED NAILFOLD CAPILLAROSCOPY PARAMETERS ARE PREDICTIVE OF SLE ONSET IN CONNECTIVE TISSUE DISEASES SUBGROUP}

${ }^{1}$ M Meroni*, ${ }^{1} \mathrm{M}$ Limonta, ${ }^{2} \mathrm{M}$ Cutolo. ${ }^{1}$ ASST Papa Giovanni XXIII, Rheumatology, Bergamo, Italy; ${ }^{2}$ University of Genova, Genova, Italy

\subsection{6/lupus-2017-000215.221}

Background and aims Nailfold capillaroscopy (NVC) is an useful, non-invasive, reproducible and cost-effective favourable diagnostic tool able to assess the shape of capillaries in the periungal region and the presence of their peculiar abnormalities, essential in the differential diagnosis of connective tissue diseases (CTD).

Methods The aim of the study was to evaluate if selected NVC pictures are linked to SLE onset in a cohort of 42
CTD-affected women presenting Raynaud's phenomenon, observed over 36 months. All of them were examined by this method every 6 months. We considered the following NVC parameters: presence of ectasic capillary loops (diameter $\geq 20 \mu \mathrm{m})$; megacapillaries $(\geq 50 \mu \mathrm{m})$; hemosiderin deposits; capillary number reduction; neo-angiogenesis phenomena; micro-vascular array disorganisation. CTD and SLE diagnoses were posed according to the 2015 ACR/SLICC criteria. Qualitative variables were expressed in frequencies; their association, by non-parametric tests. Quantitative variables were assessed by analysis of co-variance.

Results The presence of hemosiderin deposits, ectasic loops and neo-angiogenic phenomena was strongly associated to the clinical subgroup of patients that later developed SLE (12/42 subjects; $\mathrm{OR}=13.5)$. The variable meandering deposits was the more strongly associated to SLE onset $(\mathrm{OR}=8.32 ; \mathrm{p}<0.0101)$. The independent variables ectasic loops $(\mathrm{OR}=12.16)$ and neoangiogenic phenomena $(\mathrm{OR}=6.60)$ were predictive for the persistence of CTD diagnosis.

Conclusions Nailfold capillaroscopy, summarising, can help in CTD management, since the presence of typical capillaroscopic abnormalities seems to be related to the development of SLE.

\section{ASSESSMENT OF THE RISK OF FLARES IN SYSTEMIC LUPUS ERYTHEMATOSUS}

${ }^{1} \mathrm{M}$ Minodora*, ${ }^{2} \mathrm{~L}$ Mazur-Nicorici, ${ }^{3} \mathrm{~V}$ Sadovici-Bobeica, ${ }^{3} \mathrm{M}$ Garabajiu. ${ }^{1}$ State Medical and Pharmaceutical University - Nicolae Testemitanu, Department of Internal Medicine, Chisinau, Moldova; ${ }^{5}$ State Medical and Pharmaceutical University - Nicolae Testemitanu, Department of Internal Medicine Discipline Cardiology, Chisinau, Moldova; ${ }^{3}$ State Medical and Pharmaceutical University - Nicolae Testemitanu, Department of Internal MedicineDiscipline Internal Medicine, Chisinau, Moldova

\subsection{6/lupus-2017-000215.222}

Background and aims To evaluate the risc of flares in SLE patients

Methods A prospective analytic study of SLE patients (SLICC, 2012 criteria) with a fallowed-up in 5 visits: baseline visit, month 3, month 5, month 9 and month 12 visit. At visits disease activity (SLEDAI, SLAM), SELENA/SLEDAI flare index and laboratory tests were assessed.

Results The study included 102 patients, 94,1\% females, age \pm SD $42.4 \pm 13.3$ (range 20-73) years, disease duration \pm SD 93.9 \pm 77.1 (range 0,1-228) months. During fallow-up, 55 flares were enregistered, including 11 severe, with a SLEDAI increase with 3-17 points. The incidence of flares was 0,53 patient/year, the for severe flares - 0,10 patient/year. To assess the risk of flares, potential risk factors were studied (table 1).

The main risk factors were laboratory findings, while only active pulmonary involvement derived from SLAM and antiphospholipid syndrome, as clinical and laboratory variables, associated with the flares in SLE patients.

Conclusions In our study, the incidence of flare in a 12 moths period was $53,9 \%$, including $10,8 \%$ of severe flares. We have determined that patients with high ERS, low $\mathrm{Hb}$ and lymphocytopenia are at risk for flares and antiphospholipid syndrome and pulmonary involvement were the main clinical risk factors in our cohort. 


\begin{tabular}{lllll} 
Abstract 222 Table 1 & & & & \\
\hline & Correlation coefficient & $p$ & RR & $95 \%$ Cl \\
\hline ESR & 0,34 & $<0,05$ & 1,56 & $0,36-0,87$ \\
ANA & 0,22 & $>0,05$ & 1,12 & $0,43-1,50$ \\
AntiDNA Ab & 0,13 & $>0,05$ & 0,98 & $0,67-2,13$ \\
Low Hb level & 0,48 & $<0,05$ & 1,99 & $0,45-0,80$ \\
Low leucocytes & 0,23 & $>0,05$ & 1,01 & $0,56-1,33$ \\
Low lymphocytes & 0,56 & $<0,05$ & 2,05 & $0,33-0,67$ \\
Antiphospholipid syndrome & - & - & 2,30 & $0,61-0,88$ \\
Pulmonary Involvement (SLAM) & - & - & 1,88 & $0,23-0,82$ \\
\hline
\end{tabular}

223 SERUM 25-HYDROXYVITAMIN D3 LEVELS AND CLINICAL COURSE OF SYSTEMIC LUPUS ERYTHEMATOSUS : A CROSS SECTIONAL RETROSPECTIVE COHORT STUDY IN A REFERRAL CENTRE

${ }^{1,2}{ }^{2} \mathrm{M}$ Mohd Noh*, ${ }^{2} \mathrm{DM}$ Suahilai, ${ }^{2} \mathrm{NS}$ Shahril, ${ }^{3} \mathrm{SA}$ Alias. ${ }^{1}$ University Malaysia Sabah, Faculty Of Medicine andAllied Health Sciences, Kajang, Malaysia; ${ }^{2}$ Hospital Putrajaya, Medicine, Putrayaja, Malaysia; ${ }^{3}$ Hospital Putrajaya, Medical, Putrajaya, Malaysia

\subsection{6/lupus-2017-000215.223}

Background and aims To study the association between serum 25-Hydroxyvitamin D3 levels and clinical manifestation, disease activity, and disease damage of systemic lupus erythematosus (SLE).

Methods This was a retrospective cross sectional study of SLE patients seen between 1996 until 2015. Patients were grouped according to the Vitamin D3 levels : group $1(<25 \mathrm{nmol} / \mathrm{L}$ : deficiency), group $2(25-75 \mathrm{nmol} / \mathrm{L}$ : insufficiency) and group 3 (>75 nmol/L : adequate). Assessment of disease activity was done using Systemic Lupus Erythematosus Disease Activity Index Selena Modification (SLEDAI) while Systemic Lupus International Collaborating Clinics (SLICC) was use for disease damage.

Results A total of 42 patients had their serum 25-Hydroxyvitamin D3 levels taken at one point of their visit. Majority were females $(n=41)$. Mean age was 37.2 years $(S D \pm 13.13)$ and mean duration of illness 9.5 years $(\mathrm{SD} \pm 5.7)$. The proportion of patients with 25-Hydroxyvitamin D3 level group 1 was $31 \%$, group 2 was $61.9 \%$ and group 3 was $7.1 \%$ respectively. Main clinical manifestations were haematological $71.1 \%$, arthritis $68.9 \%$, malar rash $53.3 \%$. SLEDAI mild activity (0-3) $90.5 \%$, moderate activity (4-8) was $4.8 \%$ and severe activity $(>8)$ was $4.8 \%$. SLICC showed $78.6 \%$ had no damage and $21.4 \%$ with damage. Test of association using ANOVA, did not show any significance between Vitamin D3 level and SLEDAI, SLICC and clinical manifestations were observed among the group.

Conclusions Vitamin D insufficiency and deficiency was common in our SLE cohort.However, we did not find significant association between vitamin D deficiency and disease activity, damage or clinical manifestations. The study limitation includes small number of patients and retrospective design.

\section{PROTEIN-LOSING ENTEROPATHY AND FULMINANT INTESTINAL VASCULITIS IN A FILIPINO LUPUS PATIENT: A CASE REPORT}

RM Molina, S Navarra. University of Santo Tomas, Internal Medicine- Section of Rheumatology, Metro Manila, Philippines

\subsection{6/lupus-2017-000215.224}

Background and aims We describe a rare case of GI vasculitis flare in SLE presenting as diarrhoea, hematochezia and profound hypoalbuminemia.

Methods Case report

Results A 49 year old patient has had stable SLE in the past 22 years until she developed episodes of diffuse abdominal pains accompanied by alternating diarrhoea with constipation for 12 months; colonoscopy showed rectal ulcers and abdominal CT scan showed colonic diverticulosis. She received supportive therapy with only minimal relief. She was first admitted due to severe abdominal pain and worsening diarrhoea; laboratory tests disclosed thrombocytopenia, hypocomplementemia and high titer anti-dsDNA; there was dramatic resolution of symptoms with high dose corticosteroid and she was discharged significantly improved. A few weeks later while on tapering prednisone, she was re-admitted because of recurrence of profuse diarrhoea with severe electrolyte imbalance. Hospital course was marked by diarrhoea, severe hypoalbuminemia with progressive anasarca requiring intravenous albumin infusions, and episodes of massive hematochezia requiring multiple blood transfusions. Colonoscopy showed ischaemic colitis with edematous friable recto-sigmoid mucosa. Intravenous corticosteroid was increased. She underwent abdominoperineal resection with ileal resection of necrotic intestinal segments; histopath confirmed haemorrhagic gangrenous necrosis of the small intestine and colon, with small and medium vessel vasculitis and thrombosis. Although immediate post-operative course was uneventful, she succumbed a few days later to fulminant bacterial peritonitis due to anastomotic failure with extension of the bowel ischemia.

Conclusions This case illustrates the diagnostic dilemma and management challenges of lupus mesenteric vasculitis, requiring intensive monitoring for complications with aggressive supportive and disease-specific measures.

\section{GASTROINTESTINAL FLARES AMONG FILIPINO PATIENTS WITH SLE: A CASE SERIES}

RM Molina*, S Navarra. University of Santo Tomas, Internal Medicine- Section of Rheumatology, Metro Manila, Philippines

\subsection{6/lupus-2017-000215.225}

Background and aims Gastrointestinal (GI) involvement in SLE ranges from $2.2 \%-9.7 \%$, and nonspecific manifestations pose a diagnostic challenge. This series describes characteristics, treatment and outcomes of patients with GI involvement as the primary manifestation of SLE activity.

Methods Case series of 10 Filipino SLE patients with proven GI flares, seen at the Lupus Clinics of University of Santo Tomas (UST) Hospital, Manila, Philippines.

Results All 10 patients were females with mean age 31.7+9.35 SD $(19-49)$ and disease duration 6.08 \pm 7.34 SD (0 - 22) years. Most commonGI manifestations were abdominal pain (100\%), ileus (60\%), vomiting (50\%) and diarrhoea(40\%). Extra-intestinal manifestations included malar rash (70\%), arthritis (60\%), 